\title{
9 Regionalökonomische Wertschöpfungseffekte der Wiener Hochschulen
}

Die regionalökonomischen Wertschöpfungseffekte einer Hochschule und ihrer Studierenden umfassen folgende drei Bereiche. Zum ersten die direkten Beschäftigungsund Einkommenseffekte, die sich aus den Personal- und Sachausgaben der Hochschulen, den Beschäftigtenzahlen der Hochschulen sowie den Ausgaben der Studierenden innerhalb der betreffenden Region ergeben. Um diese direkten Effekte präziser zu bestimmen, wurden Faktoren wie die ungleiche Geschlechterproportion, Kaufkraftzuund -abflüsse sowie unterschiedliche Wertschöpfungseffekte von täglich pendelnden Studierenden berücksichtigt (vgl. Kapitel 7.1). Analog dazu wurden auch bei den Einkommen der Hochschulbediensteten Faktoren, die die direkten Einkommenseffekte für die Stadt Wien betreffen bzw. verzerren können, berücksichtigt (vgl. Kapitel 7.2). Diese Ausgaben von Studierenden und Hochschulbeschäftigten sowie die Beschäftigten und die Sachausgaben der Hochschulen lösen direkte Einkommens- und Umsatzeffekte aus. Da die Studierenden nicht nach Haupt- und Nebenwohnsitzfällen unterschieden werden können, ist nicht klar, inwieweit die Stadt Wien zusätzlich über den Finanzausgleich durch die aufgrund der Lokalisation der Hochschulen gestiegene Einwohnerzahl profitiert. Dieser steuerliche Aspekt wurde daher aus der Studie ausgeklammert. Die direkten Wertschöpfungseffekte werden in Kapitel 9.1 beschrieben.

Aus den wertschöpfungsrelevanten Ausgaben sowie den Sachausgaben und Investitionen der Hochschulen lassen sich zweitens indirekte Beschäftigungseffekte ableiten. Dabei handelt es sich um eine Folge der wertschöpfungsrelevanten Ausgaben, die bei Unternehmen in der Stadt Umsatzsteigerungen auslösen und somit zu einem Anstieg der Beschäftigung führen. Diese indirekten Wertschöpfungseffekte ergeben sich aus den direkten Effekten minus Vorleistungen (etwa Vorprodukte, die von Unternehmen bezogen werden) und Steuern. Sie werden in dieser Studie auf Grundlage der nationalen Input-Output-Tabelle der Statistik Austria geschätzt (Statistik Austria 2010). In der nachfolgenden Wirkungsrunde werden die durch die gestiegene Beschäftigung erwirtschafteten Einkommen wieder ausgegeben und lösen neuerliche Wertschöpfungseffekte aus, wobei diese durch die Sparquote und die Importquote abnehmen. Man spricht hier drittens von den induzierten Einkommenseffekten. Die Methodik der indirekten Beschäftigungseffekte sowie der induzierten Einkommenseffekte wird in den jeweiligen Kapiteln näher erläutert.

\subsection{Die direkten Beschäftigungs- und Einkommenseffekte der Wiener Hochschulen}

Die direkten Wertschöpfungseffekte umfassen neben den direkten Beschäftigungseffekten, die von den Wiener Hochschulen als Arbeitgeber ausgehen, all jene Investitio- 
nen und Ausgaben, die innerhalb der Stadt Wien erfolgen. Es handelt sich dabei um die Summe der Ausgaben der Studierenden (um regionale Kaufkraftströme etc. bereinigt), die Nettoeinkommen der Universitätsbediensteten (um regionale Kaufkraftströme ect. bereinigt) sowie um die allgemeinen Ausgaben der Hochschulen, sofern diese Unternehmen betreffen, die in der Stadt Wien niedergelassen sind. Diese Werte wurden in Kapitel 7 erhoben und regional zugeordnet und werden an dieser Stelle zusammengefasst. Die direkten Beschäftigungseffekte (gemessen anhand der Kopfzahl, vgl. Tabelle 9.1) der Wiener Hochschulen belaufen sich auf 31.450 Beschäftigte im wissenschaftlichen sowie im Verwaltungsbereich, wobei davon 4.289 Beschäftigte aus dem Wiener Umland nach Wien pendeln.

Die Ausgaben der Studierenden - bereinigt um Einpendler aus dem Wiener Umland sowie um Kaufkraftzu- und -abflüsse nach und von Wien - belaufen sich auf 146,4 Mio. Euro im Monat. Dies ergibt ein jährliches Ausgabenvolumen von 1.757,1 Mio. Euro. Die Nettogehälter der Angestellten, ebenfalls bereinigt um Kaufkraftzu- und -abflüsse, belaufen sich für allgemeines und wissenschaftliches Universitätspersonal auf 298,9 Mio. Euro. Dazu kommen die allgemeinen Ausgaben der Hochschulen, die für Unternehmen in der Stadt Wien 242,2 Mio. Euro betragen. Daraus ergeben sich für die Stadt Wien direkte, wertschöpfungsrelevante Ausgaben durch die Universität in Form von Investitionen, Einkommenseffekten der Bediensteten sowie Ausgaben der Studierenden in einem Umfang von 2.297,8 Mio. Euro (vgl. Tabelle 9.2). Die angegebenen Effekte durch die wissenschaftlichen Veranstaltungen der Universität Wien sind hier nicht berücksichtigt, da die errechneten Werte keine direkten Ausgaben darstellen, sondern mittels eines Input-Output-Modells des VCB indirekte und induzierte Wertschöpfungseffekte berechnet worden sind.

Tabelle 9.1: Direkte Beschäftigungseffekte der Wiener Hochschulen

\begin{tabular}{lccc}
\hline & $\begin{array}{c}\text { Wien (Hauptwohnsitz } \\
\text { und Wochenpendler) }\end{array}$ & $\begin{array}{l}\text { Wiener Umland } \\
\text { (Tagespendler) }\end{array}$ & Gesamt \\
\hline Wiss. Personal & 18.501 & 2.217 & 20.718 \\
Hab./Prof. & 3.625 & 497 & 4.122 \\
Postdocs & 1.833 & 215 & 2.048 \\
Rest & 13.044 & 1.504 & 14.548 \\
\hline Allgemeines Personal & 8.660 & 2.072 & 10.732 \\
Akademiker & 3.124 & 472 & 3.596 \\
Nichtakademiker & 5.537 & 1.599 & 7.136 \\
\hline Gesamt & $\mathbf{2 7 . 1 6 1}$ & $\mathbf{4 . 2 8 9}$ & $\mathbf{3 1 . 4 5 0}$ \\
\hline
\end{tabular}

Quellen: eigene Erhebung 2013; uni:data 2013; eigene Berechnungen. 
Tabelle 9.2: Für die Stadt Wien wertschöpfungsrelevante Investitionen, Einkommen bzw. Ausgaben der Hochschulen Wiens

\begin{tabular}{lc}
\hline & in $€$ \\
\hline Wertschöpfungsrelevantes Einkommen & 298.948 .755 \\
Allgemeines Personal & 107.183 .660 \\
Wissenschaftliches Personal & 191.765 .095 \\
\hline Ausgaben der Studierenden & 1.757 .056 .813 \\
\hline Bau-, Investitions- und Sachausgaben & 242.225 .175 \\
\hline Gesamt & $\mathbf{2 . 2 9 8 . 2 3 0 . 7 4 3}$ \\
\hline
\end{tabular}

Quellen: eigene Erhebung 2013; uni:data 2013; eigene Berechnungen.

\subsection{Die indirekten Beschäftigungseffekte der Wiener Hochschulen}

Die indirekten Beschäftigungseffekte ergeben sich aus den Wirkungen der Ausgaben der Studierenden, der Hochschulbediensteten sowie aus den Bau-, Investitions- und Sachausgaben für den städtischen Arbeitsmarkt. Zur Abschätzung der Effekte werden branchenspezifische Arbeitsmarktkoeffizienten berechnet, die aus dem Quotienten der Beschäftigten und des Umsatzes bzw. der Betriebserlöse nach der volkswirtschaftlichen Gesamtrechnung gebildet werden. Damit kann branchenspezifisch bestimmt werden, wie viele Arbeitsplätze durch 1 Mio. Euro Ausgaben geschaffen werden. Die Berechnung des Koeffizienten erfolgt auf der Grundlage sekundärstatistischer Daten der volkswirtschaftlichen Gesamtrechnung der Statistik Austria, die die branchenspezifischen Beschäftigten- und Umsatzzahlen für die gesamte Volkswirtschaft zur Verfügung gestellt hat ${ }^{1}$.

\section{Indirekte Beschäftigungseffekte durch die Studierenden}

Zur Bestimmung der indirekten Beschäftigungseffekte wurden Ausgaben von Studierenden und Universitätsbediensteten branchenspezifisch zugeordnet, wobei hier auf unterschiedliche statistische Grundlagen zurückgegriffen wurde. Die Ausgabenstruktur der Studierenden wurde mittels der Studierendensozialerhebung erhoben, die der Hochschulbediensteten wird durch die sozialstatistischen Ergebnisse der Konsumerhebung der Statistik Austria - differenziert nach Einkommensklassen - ermittelt (Statistik Austria 2010, Konsumerhebung 2009/10).

1 Auch in anderen Wertschöpfungsstudien wurde auf die volkswirtschaftlichen Sekundärstatistiken zurückgegriffen, wobei in Deutschland die Werte auch für einige wenige Bundesländer - etwa Bayern - vorliegen (vgl. Bauer 1997, Griebel und Schumacher 2002). Die Berechnungen in dieser Arbeit gehen damit von der Annahme aus, dass der nationale Arbeitsplatzkoeffizient auch auf der Ebene der Bundesländer gilt. 
Die jährlichen Ausgaben der Studierenden - 1.757,1 Mio. Euro - wurden anteilsmäßig den wichtigsten Ausgabenbereichen zugeordnet (vgl. grau markierte Zeilen in Tabelle 9.3), wobei die Ausgaben für Wohnen nicht in die Berechnung der Arbeitsplatzeffekte eingeflossen sind. Den verbliebenen sieben Ausgabenbereichen wurden Wirtschaftssektoren (NACE Rev. 2 „Viersteller“ oder ÖNACE-2008-Sektoren) zugewiesen, für die Beschäftigtenzahlen sowie Umsätze (Betriebserlöse) in der amtlichen Statistik ausgewiesen sind (Spalte 2 und 3). Für diese wurden Arbeitsplatzkoeffizienten berechnet - sowohl für die einzelnen Sektoren (Spalte 4) also auch für die Ausgabenbereiche (graue Zeilen). Die unterschiedlichen Arbeitsplatzkoeffizienten resultieren aus der unterschiedlichen Kapitalintensität der Branchen: Telekommunikation oder Reisedienstleistungen haben sehr niedrige Koeffizienten (also eine hohe Kapitalintensität), die Gastronomie ist hingegen ein sehr arbeitsintensiver Sektor, in dem ein Betriebserlös von 1 Mio. Euro einem Beschäftigtenstand von rund 17,8 Personen gegenübersteht. Im letzten Schritt der Schätzung wurden diese Koeffizienten mit den Ausgaben der jeweiligen Gruppe multipliziert.

Das Ergebnis zeigt, dass den Ausgaben der Studierenden eine Beschäftigungswirkung von rund 9.064 Beschäftigten zugerechnet werden kann. Bei den Gesamtausgaben von 1.757,1 Mio. Euro, von denen 1.170,1 Mio. Euro letztendlich in die Berechnung der indirekten Beschäftigungseffekte eingeflossen sind (Wohnkosten bleiben unberücksichtigt), ergibt sich daraus ein Gesamtkoeffizient von 7,7 Arbeitsplätzen pro ausgegebener Million Euro.

Damit liegen die berechneten Werte im Vergleich zu anderen Arbeiten in einem vergleichsweise niedrigen Schätzbereich. Die Studie zur LMU-München geht von einem Gesamtkoeffizienten von 12,2 Arbeitsplätzen pro investierter Mio. Euro aus (Bauer 1997), jene der Universität Frankfurt von 8,0 Arbeitsplätzen (Bathelt und Schamp 2002). Da hier bereits Tagespendler und Kaufkraftströme berücksichtigt worden sind und die Wohnkosten nicht in die Berechnung eingeflossen sind, handelt es sich hier mit Sicherheit um einen konservativ geschätzten - aber dennoch beträchtlichen - Beschäftigungseffekt, der von den Ausgaben der Studierenden der Wiener Hochschulen ausgeht.

\section{Indirekte Beschäftigungseffekte durch die Hochschulbediensteten}

Bei der Schätzung der indirekten Beschäftigungseffekte der Hochschulbediensteten kann nicht wie bei den Studierenden auf eine detaillierte Befragung zurückgegriffen werden. Eine Alternative, die das Ausgabenverhalten abbilden kann, sind die monatlichen Verbrauchsausgaben, die von der Statistik Austria zuletzt 2010 in den sozialstatistischen Ergebnissen der Konsumerhebung publiziert wurden (Statistik Austria 2010). Darin wurden 12 Ausgabengruppen unterschieden, deren Gewichtung allerdings nach Höhe des Einkommens differenziert ist. In Tabelle 9.4 sind in Spalte 1 diese Ausgabengruppen dargestellt, die nach Einkommensquintilen differenzierten Anteile der Ausgabengruppen sind im linken Teil der Spalten 2 bis 6 dargestellt. Im rechten Teil dieser Spalten finden sich die nach dem Verteilungsschlüssel hochgerechneten Ausgaben der Universitätsbediensteten - je nach Einkommensquintil. Der obere Teil der Tabelle zeigt die Zuordnung der sieben Einkommensgruppen der Universitätsbediensteten, die anhand ihres 
Tabelle 9.3: Indirekte Arbeitsplatzeffekte der Studierendenausgaben im WS 2011

\begin{tabular}{|c|c|c|c|}
\hline 1 & 2 & 3 & 4 \\
\hline NACE Rev. 2 Abteilung/ÖNACE 2008 & Beschäftigte & $\begin{array}{l}\text { Betriebserlöse } \\
\quad(\text { Mio. } €)\end{array}$ & $\begin{array}{c}\text { Arbeitsplatz- } \\
\text { koeffizient }\end{array}$ \\
\hline Wohnen - WS: 587,0 Mio. Euro, APK ges.: - & & \multicolumn{2}{|c|}{ Arbeitsplatzeffekt; - } \\
\hline Ernährung - WS: 371,6 Mio. Euro, APK ges.: 5,8458 & & \multicolumn{2}{|c|}{ Arbeitsplatzeffekt: $2.172,2$} \\
\hline 47 Einzelhandel (ohne Handel mit Kraftfahrzeugen) & 338.807 & 53.556 & 6,3 \\
\hline 10 H.v. Nahrungs- und Futtermitteln & 68.802 & 13.134 & 5,2 \\
\hline 11 Getränkeherstellung & 9.346 & 4.636 & 2,0 \\
\hline \multicolumn{2}{|l|}{ Kleidung - WS: 92,3 Mio. Euro, APK ges.: 6,3444 } & \multicolumn{2}{|c|}{ Arbeitsplatzeffekt: 585,7} \\
\hline 47 Einzelhandel (ohne Handel mit Kraftfahrzeugen) & 338.807 & 53.556 & 6,3 \\
\hline 14 H.v. Bekleidung & 7.924 & 994 & 8,0 \\
\hline $15 \mathrm{H} \mathrm{v}$. Leder/-waren und Schuhen & 3.791 & 700 & 5,4 \\
\hline \multicolumn{2}{|l|}{ Mobilität - WS: 119,0 Mio. Euro, APK ges.: 8,0178 } & \multicolumn{2}{|c|}{ Arbeitsplatzeffekt: 953,8} \\
\hline 49 Landverkehr & 123.571 & 15.412 & 8,0 \\
\hline \multicolumn{2}{|l|}{ Kommunikation - WS: 60,8 Mio. Euro, APK ges.: 5,2516 } & \multicolumn{2}{|c|}{ Arbeitsplatzeffekt: 319,1} \\
\hline 61 Telekommunikation & 18.246 & 6.266 & 2,9 \\
\hline 62-63 Informationsdienstleistungen & 53.296 & 7.357 & 7,2 \\
\hline \multicolumn{2}{|l|}{ Freizeit - WS: 169,9 Mio. Euro, APK ges.: 14,0713 } & \multicolumn{2}{|c|}{ Arbeitsplatzeffekt: $2.391,0$} \\
\hline 56 Gastronomie & 159.385 & 7.738 & 20,6 \\
\hline $\begin{array}{ll}\text { Herstellung, Verleih und Vertrieb von Filmen und } \\
59 \text { Fernsehprogrammen }\end{array}$ & 6.751 & 783 & 8,6 \\
\hline $\begin{array}{l}\text { Reisebüros, Reiseveranstalter, Reservierungs- } \\
79 \text { dienstleistungen }\end{array}$ & 11.057 & 4.033 & 2,7 \\
\hline S Sonst Dienstleistungen (nach ÖNACE 2008) & 4.410 & 352 & 12,5 \\
\hline \multicolumn{2}{|l|}{ Studium - WS: 142,4 Mio. Euro, APK ges.: 12,2338} & \multicolumn{2}{|c|}{ Arbeitsplatzeffekt: $1.742,5$} \\
\hline 72 Forschung und Entwicklung & 8.425 & 817 & 10,3 \\
\hline 74-75 Freiberufliche wiss. Tätigkeiten, Veterinärwesen & 15.594 & 1.155 & 13,5 \\
\hline S Sonst Dienstleistungen (nach ÖNACE 2008) & 4.410 & 352 & 12,5 \\
\hline \multicolumn{2}{|l|}{ Sonstiges - WS: 214,1 Mio. Euro, APK ges.: 4,2016 } & \multicolumn{2}{|c|}{ Arbeitsplatzeffekt: 899,6} \\
\hline Alle Sektoren & 2.682 .076 & 638.353 & 4,2 \\
\hline Gesamt - WS: 1.757,1 Mio. Euro & & Arbeitsplatze & fekt: $9.063,9$ \\
\hline
\end{tabular}

Quellen: eigene Erhebung 2013; eigene Berechnungen. Anmerk.: WS = wertschöpfungsrelevante Ausg. (vgl. Kapitel 7.1), APK ges. = Arbeitsplatzkoeffizient der ges. Ausgabengruppe 
Durchschnittswertes den Quintilen der Konsumerhebung der Statistik Austria zugeordnet wurden. Die Auswertung ermöglicht eine Schätzung der Konsumausgaben der Universitätsbediensteten - 276,2 Mio. Euro nach Abzug einer durchschnittlichen Sparquote von 7,6\% - nach den 12 Ausgabengruppen der Konsumerhebung. Um die indirekten Beschäftigungseffekte daraus zu bestimmen, werden - analog zu den Konsumausgaben der Studierenden - diese Ausgabengruppen mit branchenspezifischen Arbeitsplatzkoeffizienten gewichtet.

Die Schätzung der indirekten Beschäftigungseffekte durch die Ausgaben der Hochschulbediensteten für die Stadt Wien beläuft sich auf rund 1.745 Arbeitsplätze (vgl. Tabelle 9.5). Analog zur Schätzung der Studierendenausgaben wurden den Ausgabenbereichen ein oder mehrere Wirtschaftsbereiche zugeordnet. Von den 32,2 Mio. bei den Ausgaben für Wohnen und Energie wurden nur 44,3\% berücksichtigt, die gemäß Konsumerhebung der Statistik Austria nicht Ausgaben für Mieten, sondern nur für Energie, Wasser und erhaltende Baumaßnahmen darstellen. Der Gesamtkoeffizient liegt damit bei 7,3 Arbeitsplätzen pro ausgegebener Mio. Euro und damit geringfügig unter dem Wert der Ausgaben der Studierenden. Die Vergleichsstudien haben für München einen Gesamtkoeffizient von 11,6 (Bauer 1997, 95) und für Frankfurt von 7,2 errechnet (Griebel und Schumacher 2002, 79 f.); auch hier ist der Koeffizient für Wien als konservativ einzustufen.

\section{Beschäftigungseffekte durch die allgemeinen Ausgaben der Hochschulen}

Die allgemeinen Ausgaben der Wiener Hochschulen beliefen sich im Jahr 2011 auf 353,8 Mio. Euro, davon flossen 242,2 Mio. Euro an Unternehmen, die in Wien niedergelassen sind (vgl. Tabelle 7.7). Diese wienbezogenen allgemeinen Ausgaben betrafen zum Großteil Sachausgaben (175,8 Mio. Euro), Investitionen (23,1 Mio. Euro) und Aufwendungen für Bau- und Gebäudeerhaltung (43,3 Mio. Euro). Für diese drei Ausgabenbereiche werden ebenfalls Arbeitsplatzkoeffizienten berechnet, Basis bilden hier wieder Beschäftigtenzahlen und Betriebserlöse der Sektoren, die den drei Ausgabenbereichen zugeordnet werden. Für die drei Ausgabenbereiche der allgemeinen Ausgaben der Wiener Hochschulen wurden durch die Zusammenstellung der relevanten Branchen (vgl. Tabelle 9.6) drei gruppenspezifische Arbeitsplatzkoeffizienten errechnet, aus denen sich die indirekten Arbeitsmarkteffekte abschätzen lassen. Diese belaufen sich für die allgemeinen Ausgaben in Summe auf rund 2.067 Beschäftigte, was einem Gesamtkoeffizienten von 8,5 entspricht. 


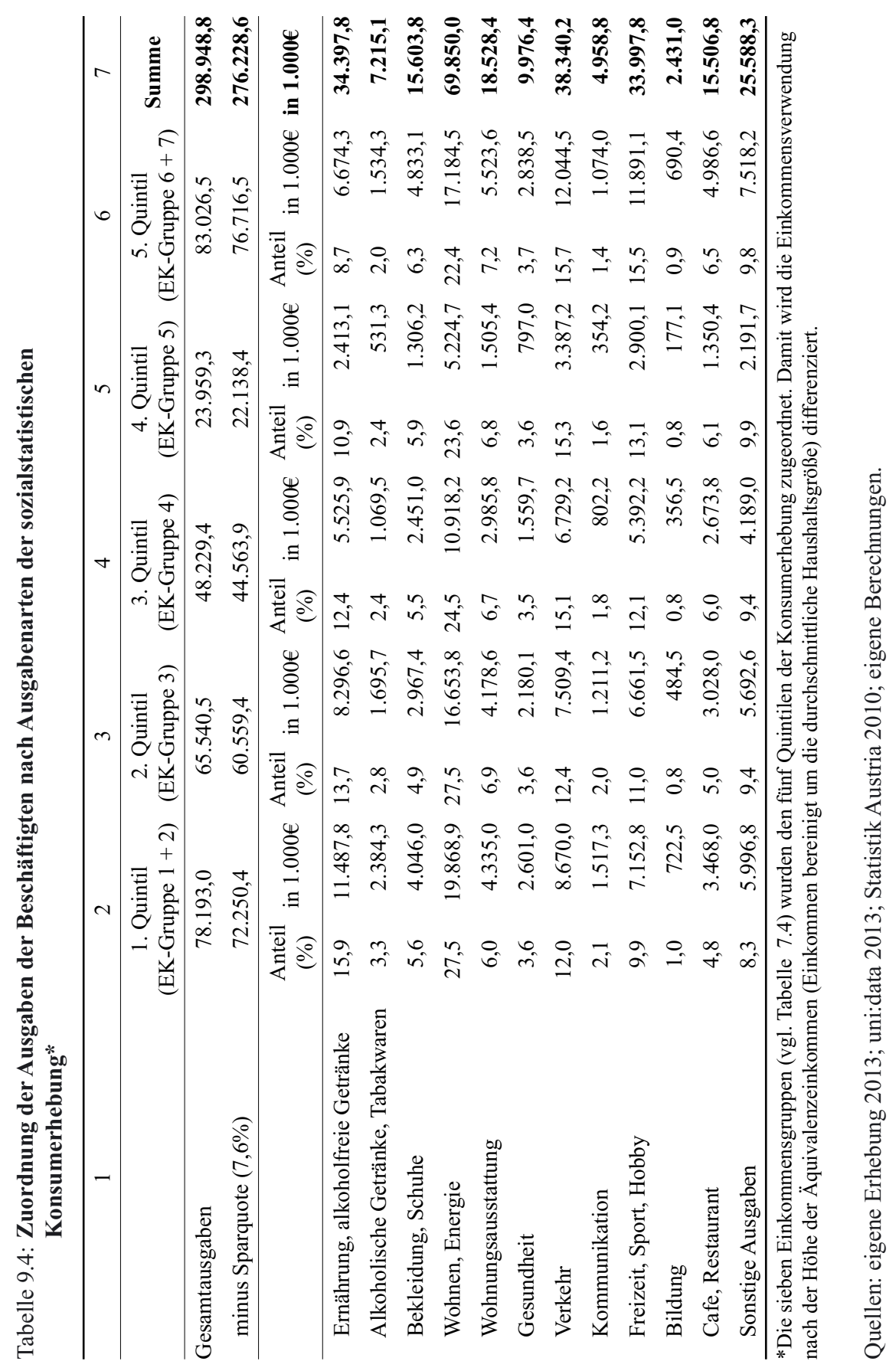


Tabelle 9.5a: Indirekte Arbeitsplatzeffekte der Ausgaben der Beschäftigten der Hochschulen Wiens 2011

\begin{tabular}{|c|c|c|c|c|}
\hline 1 & 2 & \multicolumn{2}{|c|}{3} & 4 \\
\hline NACE Rev. 2 Abteilung/ÖNACE 2008 & Beschäftigte & \multicolumn{2}{|c|}{ Betriebserlöse (Mio. €) } & AP-Koeffizient \\
\hline \multicolumn{3}{|c|}{ Ernährung, alkoholfr. Getr. - WS: 34,4 Mio. Euro, APK ges.: 5,8458 } & \multicolumn{2}{|c|}{ Arbeitsplatzeffekt 201,1 } \\
\hline 47 Einzelhandel & 338.807 & & 53.556 & 6,3 \\
\hline 10 H.v. Nahrungs- und Futtermitteln & 68.802 & & 13.134 & 5,2 \\
\hline 11 Getränkeherstellung & 9.346 & & 4.636 & 2,0 \\
\hline \multicolumn{3}{|c|}{ Alk. Getränke, Tabakwaren - WS: 7,2 Mio. Euro, APK ges.: 5,9776 } & \multicolumn{2}{|c|}{ Arbeitsplatzeffekt 43,1} \\
\hline 47 Einzelhandel & 338.807 & & 53.556 & 6,3 \\
\hline 11 Getränkeherstellung & 9.346 & & 4.636 & 2,0 \\
\hline 12 Tabakverarbeitung & 787 & & 183 & 4,3 \\
\hline \multicolumn{3}{|c|}{ Bekleidung, Schuhe - WS: 15,6 Mio. Euro, APK ges.: 6,3444 } & \multicolumn{2}{|c|}{ Arbeitsplatzeffekt 98,9} \\
\hline 47 Einzelhandel & 338.807 & & 53.556 & 6,3 \\
\hline 14 H.v. Bekleidung & 7.924 & & 994 & 8,0 \\
\hline 15 H v. Leder/-waren und Schuhen & 3.791 & & 700 & 5,4 \\
\hline \multicolumn{3}{|c|}{ Wohnen, Energie* - WS: 30,9 Mio. Euro, APK ges.: 0,6515 } & \multicolumn{2}{|c|}{ Arbeitsplatzeffekt 20,2} \\
\hline 35 Energieversorgung & 27.216 & & 29.519 & 0,9 \\
\hline 36 Wasserversorgung & 2.639 & & 541 & 4,9 \\
\hline 37 Abwasserentsorgung & 2.667 & & 639 & 4,2 \\
\hline 43 Sonst. Bautätigkeiten & 180 & & 19.497 & 9,2 \\
\hline \multicolumn{3}{|c|}{ Wohnungsausstattung - WS: 18,5 Mio. Euro, APK ges.: 6,5259 } & \multicolumn{2}{|c|}{ Arbeitsplatzeffekt 120,9} \\
\hline 31 H.v. Möbeln & 30.275 & & 3.001 & 10,1 \\
\hline 47 Einzelhandel & 338.807 & & 53.556 & 6,3 \\
\hline \multicolumn{3}{|c|}{ Gesundheit - WS: 10,0 Mio. Euro, APK ges.: 12,532 } & \multicolumn{2}{|c|}{ Arbeitsplatzeffekt 125,0} \\
\hline S Sonst. Dienstleistungen (ohne 94 u. 96) & 4.410 & & 352 & 12,5 \\
\hline \multicolumn{3}{|l|}{ Verkehr - WS: 38,3 Mio. Euro, APK ges.: 4,4052 } & \multicolumn{2}{|c|}{ Arbeitsplatzeffekt 168,9} \\
\hline 52 Dienstleistungen für den Verkehr & 49.344 & & 14.919 & 3,3 \\
\hline $45 \mathrm{Kfz}-$ Handel und -reparatur & 77.739 & & 26.568 & 2,9 \\
\hline 49 Landverkehr & 123.571 & & 15.412 & 8,0 \\
\hline
\end{tabular}

Quellen: eigene Erhebung 2013; uni:data 2013; eigene Berechnungen. 
Tabelle 9.5b: Indirekte Arbeitsplatzeffekte der Ausgaben der Beschäftigten der Hochschulen Wiens 2011 (Fortsetzung)

\begin{tabular}{|c|c|c|c|}
\hline 1 & 2 & 3 & 4 \\
\hline NACE Rev. 2 Abteilung/ÖNACE 2008 & Beschäftigte & Betriebserlöse (Mio. €) & AP-Koeffizient \\
\hline \multicolumn{2}{|c|}{ Kommunikation - WS: 5,0 Mio. Euro, APK ges.: 4,8647 } & \multicolumn{2}{|c|}{ Arbeitsplatzeffekt 24,1} \\
\hline 60 Rundfunkveranstalter & 4.964 & 1.245 & 4,0 \\
\hline 61 Telekommunikation & 18.246 & 6.266 & 2,9 \\
\hline 62 IT-Dienstleistungen & 37.298 & 4.928 & 7,6 \\
\hline \multicolumn{2}{|c|}{ Freizeit, Sport, Hobby - WS: 34,0 Mio. Euro, APK ges.: 14,3187 } & \multicolumn{2}{|c|}{ Arbeitsplatzeffekt 486,8} \\
\hline 55 Beherbergung & 105.551 & 7.149 & 14,8 \\
\hline 56 Gastronomie & 159.385 & 7.738 & 20,6 \\
\hline 59 Filmherstellung/-verleih; Kinos & 6.751 & 783 & 8,6 \\
\hline 79 Reisebüros und Reiseveranstalter & 11.057 & 4.033 & 2,7 \\
\hline S Sonst. Dienstleistungen (ohne 94 u. 96) & 4.410 & 352 & 12,5 \\
\hline \multicolumn{2}{|c|}{ Bildung - WS: 2,4 Mio. Euro, APK ges.: 11,7255 } & \multicolumn{2}{|c|}{ Arbeitsplatzeffekt 28,5} \\
\hline S Sonst. Dienstleistungen (ohne 94 u. 96) & 4.410 & 352 & 12,5 \\
\hline 74 Sonst. freiberufl./techn. Tätigkeiten & 11.044 & 868 & 12,7 \\
\hline 72 Forschung und Entwicklung & 8.425 & 817 & 10,3 \\
\hline \multicolumn{2}{|c|}{ Cafe, Restaurant - WS: 15,5 Mio. Euro, APK ges.: 20,5974 } & \multicolumn{2}{|c|}{ Arbeitsplatzeffekt 319,4} \\
\hline 56 Gastronomie & 159.385 & 7.738 & 20,6 \\
\hline \multicolumn{2}{|c|}{ Sonstige Ausgaben - WS: 25,6 Mio. Euro, APK ges.: 4,201 } & \multicolumn{2}{|c|}{ Arbeitsplatzeffekt 107,5 } \\
\hline Alle Sektoren & 2.682 .076 & 638.353 & 4,2 \\
\hline Gesamt - WS: 237,5 Mio. Euro & & Arbeitspla & zeffekt $1.744,5$ \\
\hline
\end{tabular}

Quellen: eigene Erhebung 2013; uni:data 2013; eigene Berechnungen.

Anmerkungen:

WS = wertschöpfungsrelevante Ausgaben (vgl. Tabelle 9.4)

APK ges. $=$ Arbeitsplatzkoeffizient der gesamten Ausgabengruppe

*) Von den 69,9 Mio. wertschöpfungsrelevanten Ausgaben im Bereich Wohnen und Energie (gemäß Tabelle 9.4) wurden nur 44,3 \% oder 30,9 Mio. Euro, also exklusive Wohnkosten, für die indirekten Arbeitsplatzeffekte berücksichtigt. 
Tabelle 9.6: Indirekte Arbeitsplatzeffekte der allgemeinen Ausgaben der Hochschulen Wiens 2011

\begin{tabular}{|c|c|c|c|}
\hline 1 & 2 & 3 & 4 \\
\hline NACE Rev. 2 Abteilung/ÖNACE 2008 & Beschäftigte & Betriebserlöse (Mio. €) & AP-Koeffizient \\
\hline \multicolumn{3}{|c|}{ Bau- und Gebäudeerhaltung - WS: 43,3 Mio. Euro, APK ges.: 6,8160 } & Arbeitsplatzeffekt: 295,0 \\
\hline 41 Hochbau & 64.490 & 12.948 & 5,0 \\
\hline 42 Tiefbau & 29.719 & 7.806 & 3,8 \\
\hline 43 Sonst. Bautätigkeiten & 180.142 & 19.497 & 9,2 \\
\hline \multicolumn{2}{|c|}{ Investitionen - WS: 23,1 Mio. Euro, APK ges.: 4,6829 } & & Arbeitsplatzeffekt: 108,2 \\
\hline 72 Forschung und Entwicklung & 8.425 & 817 & 10,3 \\
\hline 74 Sonst. freiberufl./techn. Tätigkeiten & 11.044 & 868 & 12,7 \\
\hline 26 H.v. Datenverarbeitungsgeräten & 20.349 & 4.922 & 4,1 \\
\hline 27 H.v. elektrischen Ausrüstungen & 40.698 & 9.491 & 4,3 \\
\hline 28 Maschinenbau & 70.447 & 16.961 & 4,2 \\
\hline 33 Reparatur/Installation v. Maschinen & 27.440 & 5.039 & 5,4 \\
\hline \multicolumn{2}{|c|}{ Sachausgaben - WS: 175,8 Mio. Euro, APK ges.: 9,4607 } & \multicolumn{2}{|c|}{ Arbeitsplatzeffekt: $1.663,6$} \\
\hline 81 Gebäudebetreuung; Gartenbau & 70.995 & 2.999 & 23,7 \\
\hline 80 Private Wach- und Sicherheitsd. & 12.387 & 362 & 34,2 \\
\hline 82 Wirtschaftliche Dienstleistungen & 15.110 & 1.727 & 8,8 \\
\hline 61 Telekommunikation & 18.246 & 6.266 & 2,9 \\
\hline 62 IT-Dienstleistungen & 37.298 & 4.928 & 7,6 \\
\hline
\end{tabular}

Quellen: eigene Erhebung 2013; eigene Berechnungen.

Anmerkungen: WS = wertschöpfungsrelevante Ausgaben (vgl. Tabelle 9.4)

APK ges. = Arbeitsplatzkoeffizient der gesamten Ausgabengruppe 
Tabelle 9.7: Indirekte Einkommenseffekte der Hochschulen Wiens 2011

\begin{tabular}{|c|c|c|c|c|}
\hline & $\begin{array}{l}\text { Wertschöp- } \\
\text { fungsrelev. } \\
\text { Ausgaben } \\
\text { (in Mio. } € \text { ) }\end{array}$ & $\begin{array}{l}\text { Arbeitsplatz- } \\
\text { effekt }\end{array}$ & $\begin{array}{l}\text { Bruttolöhne } \\
\text { in Euro je } \\
\text { unselbständig } \\
\text { Beschäftigtem }\end{array}$ & $\begin{array}{l}\text { indirekte EK- } \\
\text { Effekte in } €\end{array}$ \\
\hline \multicolumn{5}{|l|}{ Ausgaben Studierende } \\
\hline Ernährung & 371,50 & $2.171,6$ & 30.905 & 67.113 .298 \\
\hline Kleidung & 92,30 & 585,6 & 19.206 & 11.247 .034 \\
\hline Mobilität & 118,90 & 953,6 & 34.458 & 32.859 .149 \\
\hline Kommunikation & 60,70 & 319,0 & 52.610 & 16.782 .590 \\
\hline Freizeit & 169,90 & $2.390,4$ & 24.327 & 58.151 .261 \\
\hline Studium & 142,40 & $1.742,1$ & 30.199 & 52.609 .678 \\
\hline Sonstiges & 214,10 & 899,4 & 37.690 & 33.898 .386 \\
\hline Gesamt Studierende (inkl. Wohnen) & 1756,60 & $9.061,6$ & & 272.661.395 \\
\hline \multicolumn{5}{|l|}{ Ausgaben Universitätsbedienstete } \\
\hline Ernährung, alkoholfreie Getränke & 34,4 & 201,1 & 30.905 & 6.214 .474 \\
\hline Alkoholische Getränke, Tabakwaren & 7,2 & 43,1 & 42.799 & 1.845 .887 \\
\hline Bekleidung, Schuhe & 15,6 & 99,0 & 19.206 & 1.901 .314 \\
\hline Wohnen, Energie (exkl. Mietkosten) & 30,9 & 20,2 & 44.716 & 901.447 \\
\hline Wohnungsausstattung & 18,5 & 120,9 & 23.393 & 2.828 .553 \\
\hline Gesundheit & 10,0 & 125,0 & 26.532 & 3.317 .130 \\
\hline Verkehr & 38,3 & 168,9 & 37.250 & 6.291 .410 \\
\hline Kommunikation & 5,0 & 24,1 & 57.365 & 1.383 .830 \\
\hline Freizeit, Sport, Hobby & 34,0 & 486,8 & 24.030 & 11.697 .875 \\
\hline Bildung & 2,4 & 28,5 & 36.090 & 1.028 .755 \\
\hline Cafe, Restaurant & 15,5 & 319,4 & 13.839 & 4.420 .192 \\
\hline Sonstige Ausgaben & 25,6 & 107,5 & 37.690 & 4.052 .076 \\
\hline Gesamt Universitätsbedienstete & 237,5 & $1.744,5$ & & 45.882.943 \\
\hline \multicolumn{5}{|l|}{ Allgemeine Ausgaben Universität } \\
\hline Bau- und Gebäudeerhaltung & 6,1 & 295,0 & 38.744 & 11.428 .905 \\
\hline Investitionen & 13,0 & 108,2 & 48.634 & 5.262 .950 \\
\hline Sachausgaben & 160,6 & $1.663,6$ & 35.967 & 59.833 .180 \\
\hline Gesamt allgemeine Ausgaben & 179,8 & $2.066,8$ & & 76.525 .035 \\
\hline Gesamt & & & & 395.069 .373 \\
\hline
\end{tabular}

Quellen: eigene Erhebung 2013; uni:data 2013; Statistik Austria; eigene Berechnungen. 
Tabelle 9.8: Induzierte Wertschöpfung und Beschäftigung der Hochschulen Wiens 2011

\begin{tabular}{|c|c|c|c|c|c|}
\hline & $\begin{array}{l}\text { indirekte } \\
\text { EK-Effekte } \\
\quad \text { in } €\end{array}$ & $\begin{array}{l}\text { Netto } \\
\text { Wertschöp- } \\
\text { fungsmulti- } \\
\text { plikator }\end{array}$ & $\begin{array}{l}\text { Beschäfti- } \\
\text { gungsmulti- } \\
\text { plikator }\end{array}$ & $\begin{array}{l}\text { Induzierte } \\
\text { Wertschöp- } \\
\text { fung in } €\end{array}$ & $\begin{array}{l}\text { Induzierte } \\
\text { Beschäftigte }\end{array}$ \\
\hline \multicolumn{6}{|l|}{ Ausgaben Studierende } \\
\hline Ernährung & 67.113 .298 & 0,5185 & 0,0099 & 34.798 .245 & 664 \\
\hline Kleidung & 11.247 .034 & 0,2740 & 0,0108 & 3.081 .687 & 122 \\
\hline Mobilität & 32.859 .149 & 0,6632 & 0,0121 & 21.792 .187 & 398 \\
\hline Kommunikation & 16.782 .590 & 0,6584 & 0,0202 & 11.049 .657 & 339 \\
\hline Freizeit & 58.151 .261 & 0,6391 & 0,0103 & 37.164 .471 & 599 \\
\hline Studium & 52.609 .678 & 0,7269 & 0,0113 & 38.241 .975 & 595 \\
\hline Sonstiges & 33.898 .386 & 0,4948 & 0,0092 & 16.772 .921 & 312 \\
\hline Gesamt Studierende & 272.661.395 & & & 162.901.144 & 3.029 \\
\hline \multicolumn{6}{|l|}{ Ausgaben Universitätsbedienstete } \\
\hline Ernährung, alkoholfreie Getränke & 6.214 .474 & 0,5185 & 0,0099 & 3.222 .205 & 62 \\
\hline Alk. Getränke, Tabakwaren & 1.845 .887 & 0,4443 & 0,0088 & 820.127 & 16 \\
\hline Bekleidung, Schuhe & 1.901 .314 & 0,2740 & 0,0108 & 520.960 & 21 \\
\hline Wohnen, Energie (exkl. Mietk.) & 901.447 & 0,5595 & 0,0068 & 504.360 & 6 \\
\hline Wohnungsausstattung & 2.828 .553 & 0,5866 & 0,0134 & 1.659 .229 & 38 \\
\hline Gesundheit & 3.317 .130 & 0,7528 & 0,0116 & 2.497 .135 & 38 \\
\hline Verkehr & 6.291 .410 & 0,6762 & 0,0115 & 4.254 .252 & 72 \\
\hline Kommunikation & 1.383 .830 & 0,5404 & 0,0069 & 747.821 & 10 \\
\hline Freizeit, Sport, Hobby & 11.697 .875 & 0,6391 & 0,0103 & 7.476 .112 & 120 \\
\hline Bildung & 1.028 .755 & 0,7269 & 0,0113 & 747.802 & 12 \\
\hline Cafe, Restaurant & 4.420 .192 & 0,7565 & 0,0111 & 3.343 .875 & 49 \\
\hline Sonstige Ausgaben & 4.052 .076 & 0,4948 & 0,0092 & 2.004 .967 & 37 \\
\hline Gesamt Universitätsbedienstete & 45.882 .943 & & & 21.071.965 & 339 \\
\hline \multicolumn{6}{|l|}{ Allgemeine Ausgaben Universität } \\
\hline Bau- und Gebäudeerhaltung & 11.428 .905 & 0,5534 & 0,0094 & 6.324 .756 & 107 \\
\hline Investitionen & 5.262 .950 & 0,3315 & 0,0077 & 1.744 .668 & 41 \\
\hline Sachausgaben & 59.833 .180 & 0,6679 & 0,0102 & 39.962 .581 & 610 \\
\hline Gesamt allgemeine Ausgaben & 76.525.035 & & & 74.452 .812 & 1.183 \\
\hline Gesamt & 395.069 .373 & & & 258.425.921 & 4.550 \\
\hline
\end{tabular}

Quellen: eigene Erhebung 2013; uni:data 2013; Statistik Austria; eigene Berechnungen. 


\subsection{Induzierte Wertschöpfungseffekte der Hochschulen Wiens}

Aus den indirekten Beschäftigungseffekten lassen sich wieder Einkommenseffekte ableiten, die durch die Löhne der geschaffenen Arbeitsplätze entstehen. Dazu müssen die in Kapitel 9.2 geschätzten Beschäftigungseffekte der verschiedenen, durch die Hochschulen ausgelösten Ausgaben (Ausgaben der Studierenden, Ausgaben der Universitätsbediensteten, allgemeine Ausgaben der Universität), mit den Bruttolöhnen je Beschäftigten der jeweiligen Branchen hochgerechnet werden (vgl. Bauer 1997, 97, Griebel und Kobiela 2002, 96, Stoetzer und Krähmer 2007, 33)². Für die unterschiedlichen Ausgabenarten wurden - analog zu den Arbeitsplatzeffekten - die durchschnittlichen Bruttolöhne der jeweiligen Branchen berechnet zu den indirekten Einkommenseffekten hochgerechnet (vgl. Tabelle 9.7, Spalte Bruttolöhne). Dabei zeigt sich eine gewisse Umkehr der unterschiedlichen Arbeitsplatzeffekte: Branchen mit einem hohen Arbeitsmarkteffekt (also einer hohe Beschäftigtenzahl je Mio. Euro) weisen niedrige Bruttolöhne auf, wodurch die indirekten Einkommenseffekte verhältnismäßig gering ausfallen. Die Gastronomiebranche ist dafür ein sehr gutes Beispiel.

Diese Berechnung hat ergeben, dass die indirekten Beschäftigungseffekte ein jährliches Einkommen von 395,1 Mio. Euro induzieren, wobei die indirekten Einkommenseffekte der Studierenden mit 272,7 Mio. Euro den größten Anteil ausmachen (vgl. Tabelle 9.7). Um die daraus entstehenden Wertschöpfungseffekte, die gewissermaßen in einer zweiten Wirkungs- bzw. Ausgabenrunde entstehen, abschätzen zu können, werden von Wertschöpfungsstudien zwei unterschiedliche Methoden angewendet: erstens die makroökonomische oder keynesianistische Multiplikatoranalyse, die die Wertschöpfung einer induzierten Einkommensgröße abschätzt [eine detaillierte Beschreibung findet sich in den Arbeiten von Griebel und Kobiela (2002) sowie von Bauer (1997), ein vereinfachtes Multiplikatormodell beschreiben Glückler und König (2011, 345)]. Zentrale Variablen sind hier die Konsumquote, die zur Erhöhung der Wertschöpfung führt, sowie die Importquote, die aufgrund der extern erbrachten Vorleistungen die Wertschöpfung der zusätzlichen Einkommen reduziert. Auch wohlfahrtsstaatliche Transfers und Steuern führen zu einer Reduktion der regionalen Einkommen bzw. der dadurch induzierten Wertschöpfung. Es handelt sich bei der Multiplikatoranalyse um ein relativ einfaches regionales Nachfragemodell, das durch die genannten Faktoren - Konsumquote, Importquote, Steuer- und Transferquote - gebildet wird, wobei allerdings in den regionalen Wertschöpfungsstudien der Universitäten häufig auf makroökonomische Variablen zurückgegriffen wird. Ein gewisser Nachteil der Methode ergibt sich aus dem Umstand, dass branchenspezifische Aussagen nicht möglich sind (Leusing 2007, 10).

Die zweite Methode zur Schätzung der durch die indirekten Einkommen ausgelösten Wertschöpfung ist die Input-Output-Analyse (Stoetzer und Krähmer 2007, 34). Dabei wird für zahlreiche Sektoren und Güter der Zusammenhang zwischen Vorleistungen und Endprodukt hergestellt, womit regionalökonomische Effekte auch zwischen den

2 Indirekte Einkommenseffeke $=$ Arbeitsplatzeffekt $*$ Bruttolöhne/unselbständig Beschäftigte. 
Branchen festgestellt werden können. Da dazu sehr komplexe Verflechtungen zwischen Gütern und Branchen erhoben werden müssen, greifen entsprechende Studien in der Regel auf nationale Input-Output-Tabellen zurück, die die große Zahl an Branchen auf einige wenige reduzieren und damit an die regionalen Gegebenheiten anpassen (vgl. Rosner und Weinmann 2003 oder Niermann 1996).

Um die induzierten Effekte der indirekten Einkommens- und Beschäftigungseffekte in den nachfolgenden Wirtschaftsstufen zu schätzen, wird hier auf die entsprechenden Multiplikatoren der Input-Output-Tabelle der Statistik Austria zurückgegriffen, die sich auf die Verflechtungen und Wertschöpfungseffekte der österreichischen Volkswirtschaft bezieht (Statistik Austria 2012). Darin besteht eine gewisse Vereinfachung, da Vorleistungen bzw. Importe aus den restlichen Bundesländern nicht berücksichtigt werden können. Umgekehrt erlaubt es die nationale Input-Output-Tabelle, auf die branchenspezifische Struktur der Einkommenseffekte Rücksicht zu nehmen. Damit ist es möglich, für die den Ausgabearten zugeordneten Branchen der NACE Rev.2-Klassifikation den Netto-Wertschöpfungsmultiplikator sowie den Beschäftigungsmultiplikator der InputOutput-Tabelle der Statistik Austria zu erheben. Daraus lassen sich für jede Ausgabenart in Tabelle 9.8 entsprechende Multiplikatoren erheben, aus denen eine induzierte Wertschöpfung sowie eine induzierte Beschäftigung berechnet werden kann, die sich aus den oben berechneten indirekten Einkommenseffekten ableiten lassen.

Die 272,6 Mio. Euro indirekter Einkommenseffekte der Ausgaben der Studierenden induzieren in einer weiteren Wirkungsrunde jährlich 162,9 Mio. Euro Wertschöpfung und einen weiteren Beschäftigtenanstieg um 3.029 Vollzeitbeschäftigte. Summiert man die Ausgaben der Studierenden, der Universitätsbediensteten sowie der allgemeinen Ausgaben der Hochschulen, so ergibt sich nach Anwendung der Input-Output-Multiplikatoren ein induzierter jährlicher Wertschöpfungseffekt von 258,4 Mio. Euro und rund 4.550 Beschäftigten. 